\title{
Spherical Shells of Classical Gauge Field and Their Topological Charge as a Perturbative Expansion
}

\author{
Edward Farhi* \\ Center for Theoretical Physics \\ Laboratory for Nuclear Science and \\ Department of Physics \\ Massachusetts Institute of Technology \\ Cambridge, MA 02139 \\ Valentin V. Khoze ${ }^{\dagger}$ \\ Stanford Linear Accelerator Center \\ Stanford University \\ Stanford, CA 94309 \\ Krishna Rajagopal ${ }^{\ddagger}$ \\ Lyman Laboratory of Physics \\ Harvard University \\ Cambridge, MA 02138 \\ Robert Singleton, Jr. ${ }^{\S}$ \\ Department of Physics \\ Boston University \\ Boston, MA 02215
}

CTP\#2287

SLAC-PUB-6419(T)

March 1994

HUTP-94-A003

Submitted to Physical Review D

BUHEP-94-6

Typeset in REVTEX

\footnotetext{
*farhi@mitlns.mit.edu. Research supported in part by the U.S. Department of Energy (D.O.E.) under contract \#DE-AC02-76ER03069

†vkhoze@slacvm.slac.stanford.edu. Research supported in part by the D.O.E. under contract \#DE-AC03-76SF0015.

${ }^{\ddagger}$ rajagopal@huhepl.harvard.edu. Junior Fellow, Harvard Society of Fellows. Research supported in part by the National Science Foundation under grant PHY-92-18167.

§bobs@pth3.bu.edu. Research supported in part by the D.O.E. under contract \#DE-FG0291ER40676 and by the Texas National Research Laboratory Commission under grant RGFY93-278.
} 


\begin{abstract}
We consider the classical equations of motion of $S U(2)$ gauge theory, without a Higgs field, in Minkowski space. We work in the spherical ansatz and develop a perturbative expansion in the coupling constant $g$ for solutions which in the far past look like freely propagating spherical shells. The topological charge $Q$ of these solutions is typically non-integer. We then show that $Q$ can be expressed as a power series expansion in $g$ which can be nonzero at finite order. We give an explicit analytic calculation of the order $g^{5}$ contribution to $Q$ for specific initial pulses. We discuss the relation between our findings and anomalous fermion number violation, and speculate on the physical implications of our results.
\end{abstract}




\section{INTRODUCTION}

Fermion number is not conserved in the standard model [1], yet reliable techniques for calculating fermion number violating rates in high energy collisions have not been fully developed. At very low energy where the processes are best described as tunnelling events, the calculational methods [1] make use of solutions to the Euclidean field equations instantons [2]. At energies comparable to but below the sphaleron barrier [3], Euclidean methods [4 and other methods in which part of the calculation is done in Euclidean space [5] have also been applied. However, at energies well above the tunnelling barrier, it may be more appropriate to work directly in Minkowski space [6.7]. To this end, we are investigating solutions to the Minkowski space classical equations of motion. In this paper, we study $S U(2)$ gauge theory without the Higgs field.

Working in the (spatial) spherical ansatz, we explore solutions which have the property that in the far past they describe freely propagating incoming shells of energy. We do this by developing a perturbative expansion in the coupling $g$ which can be used to systematically solve the equations of motion once arbitrary initial profiles have been specified. We discuss the topological charge of these solutions, which we also develop in a power series expansion in $g$. We show that the topological charge is nonzero at order $g^{5}$. We then investigate the associated anomalous fermion production in the presence of these solutions.

Recently [7], it was shown that in the spherical ansatz the classical equations of motion of $S U(2)$ gauge theory can be reduced to two coupled nonlinear partial differential equations of motion for two gauge invariant functions of $r=|\mathbf{x}|$ and $t$. In Ref. [7], new solutions to these equations were also presented which have much in common with the previously discovered solutions [8] of Lüscher and Schechter (LS). As we will show here these explicit solutions are examples of a wide class of finite energy solutions all of which have certain general features in common. At early times they depict a thin spherical shell of energy imploding towards the origin at near the speed of light. At intermediate times the region around the origin is energetically excited and at late times the shell is expanding outward, asymptotically approaching the speed of light. 
We are particularly interested in solutions which look free in the far past. This is because our ultimate goal is to connect the classical solutions to the quantum mechanical description of the scattering of quanta which in the far past are well separated and non-interacting. For a classical solution to be said to be free in the far past we require that there exists a gauge in which the commutator term in $F_{\mu \nu}$ (see 1.3) can be neglected in the far past. This means that the theory linearizes. We also want $A_{\nu}$ in this gauge to be independent of $g$ in the far past. This means that the initial value data can be specified without reference to $g$. The LS solutions and the solutions of Ref. [7] do not satisfy this requirement. However, in this paper we will show, in the spherical ansatz, how to specify initial data which do meet this criterion.

Returning to the description of the shells, if we focus on the region near the origin we see that before the front reaches it, it is devoid of energy, i.e. it is a vacuum configuration. The energy shell excites this region and then leaves so that at late times the region is once again a vacuum configuration. However, the late time vacuum structure of this region need not coincide with its early time structure. As we now explain, the region can be associated with a local topological charge which is typically nonvanishing, non-integer, and $g$-dependent.

We define the local topological charge inside a sphere of radius $R$ as

$$
Q(R)=\frac{g^{2}}{32 \pi^{2}} \int_{-\infty}^{\infty} d t \int_{R} d^{3} x \epsilon^{\mu \nu \alpha \beta} \operatorname{Tr}\left(F_{\mu \nu} F_{\alpha \beta}\right)
$$

where the spatial integration is over the interior of the sphere. For the LS solutions, $Q(R)$ approaches a fixed value as $R$ goes to infinity whereas for the solutions of Ref. [7], $Q(R)$ grows without bound. Let us restrict our attention to all those solutions for which $Q(R)$ has a limit as $R$ goes to infinity. In this case, as $R$ goes to infinity, $Q(R)$ approaches the topological charge $Q$ defined by

$$
Q=\frac{g^{2}}{32 \pi^{2}} \int d^{4} x \epsilon^{\mu \nu \alpha \beta} \operatorname{Tr}\left(F_{\mu \nu} F_{\alpha \beta}\right),
$$

where the integration is over all of space-time.

The topological charge $Q$ can take any value, not just integer values [7]. At first sight this may appear to contradict the observation that (1.2) is a topological invariant of the field 
configuration. Let us review the usual argument [9] that leads to integer topological charge. Imagine that the region of space-time where the energy density is nonzero is bounded. Surround this region with a three dimensional surface which is topologically $S^{3}$. On this surface $F_{\mu \nu}=0$ so the gauge field is pure gauge, i.e. $A_{\mu}=(i / g) U \partial_{\mu} U^{\dagger}$ where $U$ takes values in $S U(2)$. This defines a map from $S^{3}$ into $S U(2)$ which is characterized by an integer since $\pi_{3}(S U(2))=Z$. The integrand in (1.2) is a total divergence so $Q$ can be written as an integral over the surrounding three dimensional surface. This integral computes the winding number of the map from $S^{3}$ into $S U(2)$ and therefore $Q$ is an integer.

This argument assumes that the space-time region where the energy density is nonzero is bounded. For solutions to the equations of motion, energy is conserved and the energy computed on any equal time surface is non-vanishing. It is therefore impossible to surround the space-time region of nonzero energy density with a three sphere. We have no reason to expect and we do not find integer values of $Q$.

If the topological argument applied and $Q$ were an integer then certainly $Q$ would be independent of the gauge coupling $g$. We now examine another commonly used argument which, when applicable, says that $Q$ is independent of $g$. The field strength $F_{\mu \nu}$ is defined as

$$
F_{\mu \nu}=\partial_{\mu} A_{\nu}-\partial_{\nu} A_{\mu}-i g\left[A_{\mu}, A_{\nu}\right]
$$

and the equation of motion is

$$
D^{\mu} F_{\mu \nu}=\partial^{\mu} F_{\mu \nu}-i g\left[A^{\mu}, F_{\mu \nu}\right]=0
$$

We can redefine the fields so that the equation of motion is independent of $g$. Let

$$
\begin{gathered}
A_{\mu}=\frac{1}{g} A_{\mu}^{\prime}, \\
F_{\mu \nu}=\frac{1}{g} F^{\prime}{ }_{\mu \nu}=\frac{1}{g}\left(\partial_{\mu} A^{\prime}{ }_{\nu}-\partial_{\nu} A^{\prime}{ }_{\mu}-i\left[A^{\prime}{ }_{\mu}, A^{\prime}{ }_{\nu}\right]\right) .
\end{gathered}
$$

Now the equation of motion reads 


$$
\partial^{\mu} F^{\prime}{ }_{\mu \nu}-i\left[A^{\prime \mu}, F_{\mu \nu}^{\prime}\right]=0
$$

which contains no $g$. The topological charge (1.2) is seen to be independent of $g$ :

$$
Q\left[A^{\prime}{ }_{\mu}\right]=\frac{1}{32 \pi^{2}} \int d^{4} x \epsilon^{\mu \nu \alpha \beta} \operatorname{Tr}\left(F_{\mu \nu}^{\prime} F_{\alpha \beta}^{\prime}\right)
$$

In this sense solutions to the equation of motion yield a topological charge independent of the coupling.

However, we are interested in solving (1.4) in Minkowski space with fixed initial value data which does not depend on the coupling $g$. That is, we wish to solve (1.4) specifying the field $A_{\mu}$ and its time derivative $\dot{A}_{\mu}$ at some fixed time $T$. We specify

$$
\begin{aligned}
& A_{\mu}(\mathbf{x}, T)=A_{\mu}^{\text {init }}(\mathbf{x}), \\
& \dot{A}_{\mu}(\mathbf{x}, T)=V_{\mu}^{\text {init }}(\mathbf{x}),
\end{aligned}
$$

where $A_{\mu}^{\text {init }}(\mathbf{x})$ and $V_{\mu}^{\text {init }}(\mathbf{x})$ are arbitrary functions consistent with the constraints. In terms of rescaled variables

$$
\begin{aligned}
& A_{\mu}^{\prime}(\mathbf{x}, T)=g A_{\mu}^{\mathrm{init}}(\mathbf{x}) \\
& \dot{A}_{\mu}^{\prime}(\mathbf{x}, T)=g V_{\mu}^{\mathrm{init}}(\mathbf{x})
\end{aligned}
$$

If we fix $A_{\mu}^{\text {init }}(\mathbf{x})$ and $V_{\mu}^{\text {init }}(\mathbf{x})$ then $A^{\prime}{ }_{\mu}$ actually depends on $g$. Accordingly $Q$ depends on $g$.

As an example, if $g$ is equal to zero, we are solving (1.7) with the condition that $A^{\prime}{ }_{\mu}$ and $\dot{A}^{\prime}{ }_{\mu}$ are zero at $T$. The solution is $A^{\prime}{ }_{\mu}(\mathbf{x}, t)=0$ which yields zero topological charge in (11.8). Another way to see this is to work with $g=0$ directly. The equation of motion (1.3) is

$$
\partial^{\mu}\left(\partial_{\mu} A_{\nu}-\partial_{\nu} A_{\mu}\right)=0
$$

which we can solve with fixed initial value data, yielding non-trivial solutions. In this case $Q$ given by (1.2) is seen to be zero since $g$ is zero. Thus if we solve the classical equations of motion with fixed, $g$-independent, initial value data we expect the topological charge to vanish as $g$ goes to zero. This suggests that we can develop a power series expansion in $g$ for $Q$. 
To conclude this introduction, we describe the organization of the rest of the paper. In section II we review the spherical ansatz and write the equations of motion in terms of two gauge invariant variables. In section III we show that in the spherical ansatz, the theory linearizes in the far past and the far future. We express the requirement that $A^{\mu}$ be $g_{\text {- }}$ independent in the far past in terms of the gauge invariant variables. In section IV we show how to solve the field equations as a perturbative expansion in $g$ for arbitrary initial pulses, and perform the calculation explicitly to zeroth and first order in g. In section $\mathrm{V}$ we return to our discussion of the topological charge of the solutions. We show how to expand $Q$ as a power series in $g$. We present examples of initial data for which the topological charges of the corresponding solutions are nonzero at order $g^{5}$. In appendix A, without restricting to

the spherical ansatz, we show that in source-free electromagnetism the topological charge of localized pulses vanishes. In section VI we discuss anomalous fermion number violation in the presence of classical gauge field sources. We show that the number of fermions produced, in a quantum average sense, is $Q$. In section VII, we remark upon the implications of our results for anomalous fermion number violation in high energy scattering in the Standard Model, and discuss open questions.

\section{THE SPHERICAL ANSATZ}

Here we review the spherical ansatz [10] for $S U(2)$ gauge theory without a Higgs field and show how the equations can be reduced to two equations for two gauge invariant functions. The spherical ansatz is given by expressing the gauge field $A_{\mu}$ in terms of four functions $a_{0}, a_{1}, \alpha$ and $\gamma$ of $r$ and $t$ :

$$
\begin{aligned}
& A_{0}(\mathbf{x}, t)=\frac{1}{2} a_{0}(r, t) \boldsymbol{\sigma} \cdot \hat{\mathbf{x}} \\
& A_{i}(\mathbf{x}, t)=\frac{1}{2}\left[a_{1}(r, t) \boldsymbol{\sigma} \cdot \hat{\mathbf{x}} \hat{x}_{i}+\frac{\alpha(r, t)}{r}\left(\sigma_{i}-\boldsymbol{\sigma} \cdot \hat{\mathbf{x}} \hat{x}_{i}\right)+\frac{\gamma(r, t)}{r} \epsilon_{i j k} \hat{x}_{j} \sigma_{k}\right]
\end{aligned}
$$

where $\hat{\mathbf{x}}$ is the unit three-vector in the radial direction. Note that we do not introduce $1 / g$ factors in (2.1) as was done in Refs. [10] and [0]. The action 


$$
S=-\frac{1}{2} \int d^{4} x \operatorname{Tr}\left(F_{\mu \nu} F^{\mu \nu}\right)
$$

in the spherical ansatz takes the form of an Abelian Higgs model in curved space [10],

$$
S=4 \pi \int d t \int_{0}^{\infty} d r\left[-\frac{1}{4} r^{2} f_{\mu \nu} f^{\mu \nu}-\left(D_{\mu} \chi\right)^{*} D^{\mu} \chi-\frac{g^{2}}{2 r^{2}}\left(|\chi|^{2}-\frac{1}{g^{2}}\right)^{2}\right]
$$

where

$$
f_{\mu \nu}=\partial_{\mu} a_{\nu}-\partial_{\nu} a_{\mu}
$$

is the field strength,

$$
\chi=\alpha+i\left(\gamma-\frac{1}{g}\right)
$$

is a complex scalar and

$$
D_{\mu} \chi=\left(\partial_{\mu}-i g a_{\mu}\right) \chi
$$

is the covariant derivative. The indices are raised and lowered with the $1+1$ dimensional metric $d s^{2}=-d t^{2}+d r^{2}$. (Note that in this paper we raise and lower indices with a flat space metric; we do not absorb the explicit factors of $r$ into a curved space metric as was done in Ref. [7].)

The equations of motion for the reduced theory are

$$
-\partial^{\mu}\left(r^{2} f_{\mu \nu}\right)=i g\left[\left(D_{\nu} \chi\right)^{*} \chi-\chi^{*} D_{\nu} \chi\right]
$$

and

$$
\left[-D^{2}+\frac{1}{r^{2}}\left(g^{2}|\chi|^{2}-1\right)\right] \chi=0 .
$$

It is convenient to write the complex field $\chi$ in polar form

$$
\chi(r, t)=-i \rho(r, t) \exp [i g \varphi(r, t)]
$$

which casts the equations of motion in terms of $\rho, \varphi$ and $a_{\mu}$ as 


$$
\begin{gathered}
\partial^{\mu}\left(r^{2} f_{\mu \nu}\right)+2 g^{2} \rho^{2}\left(\partial_{\nu} \varphi-a_{\nu}\right)=0, \\
\partial_{\mu} \partial^{\mu} \rho-g^{2} \rho\left(\partial^{\mu} \varphi-a^{\mu}\right)\left(\partial_{\mu} \varphi-a_{\mu}\right)-\frac{1}{r^{2}}\left(g^{2} \rho^{2}-1\right) \rho=0,
\end{gathered}
$$

and

$$
\partial^{\mu}\left[\rho^{2}\left(\partial_{\mu} \varphi-a_{\mu}\right)\right]=0 .
$$

Note that (2.9d) follows from (2.9a) so there are three, not four, independent equations of motion which is expected because of the residual $U(1)$ gauge invariance.

In $1+1$ dimensions $f_{\mu \nu}$ must be proportional to $\epsilon_{\mu \nu}$ so we can define the gauge invariant field $\psi$ through

$$
r^{2} f_{\mu \nu}=-2 \epsilon_{\mu \nu} \psi
$$

(Here $\left.\epsilon_{01}=+1.\right)$ Equation (2.9a) now becomes

$$
\partial^{\alpha} \psi=-g^{2} \epsilon^{\alpha \nu} \rho^{2}\left(\partial_{\nu} \varphi-a_{\nu}\right),
$$

which implies

$$
\partial_{\mu}\left(\frac{\partial^{\mu} \psi}{\rho^{2}}\right)-\frac{2 g^{2}}{r^{2}} \psi=0 .
$$

Substituting (2.11) into (2.9b) gives

$$
\partial_{\mu} \partial^{\mu} \rho+\frac{1}{g^{2} \rho^{3}} \partial_{\mu} \psi \partial^{\mu} \psi-\frac{1}{r^{2}}\left(g^{2} \rho^{2}-1\right) \rho=0 .
$$

Equations (2.12a) and (2.12b) are the equations of motion for the gauge invariant variables $\rho$ and $\psi$. If a gauge is chosen then the gauge-variant variables $\varphi$ and $a_{\mu}$ can be determined using (2.11). For example, in the $\varphi=0$ gauge, given $\rho$ and $\psi$, equation (2.11) directly determines $a_{\nu}$. Equations (2.12a) and (2.12b) for $\psi$ and $\rho$ can be solved after specifying initial value data, that is the values of $\psi, \dot{\psi}, \rho$ and $\dot{\rho}$ at some fixed time. Any initial value data expressed in terms of $\psi, \dot{\psi}, \rho$ and $\dot{\rho}$ will be consistent with the Gauss's law 
constraint. This is because Gauss's law is the $\nu=0$ component of equation (2.9a) which is equivalent to the $\alpha=1$ component of (2.11).

Using the equations of motion, the energy associated with the action (2.2) can be written in terms of $\rho$ and $\psi$ as

$$
E=8 \pi \int_{0}^{\infty} d r\left[\frac{1}{2}\left(\partial_{t} \rho\right)^{2}+\frac{1}{2}\left(\partial_{r} \rho\right)^{2}+\frac{1}{2 g^{2} \rho^{2}}\left(\partial_{t} \psi\right)^{2}+\frac{1}{2 g^{2} \rho^{2}}\left(\partial_{r} \psi\right)^{2}+\frac{\psi^{2}}{r^{2}}+\frac{\left(g^{2} \rho^{2}-1\right)^{2}}{4 g^{2} r^{2}}\right] .
$$

From this expression we see that the field configuration with zero energy has $\psi=0$ and $\rho=1 / g$

It is convenient to introduce a shifted field $\delta$ defined by

$$
2 g \delta=1-g^{2} \rho^{2}
$$

Equations (2.12) now become

$$
\partial_{\mu} \partial^{\mu} \psi-\frac{2}{r^{2}} \psi=-2 g \partial_{\mu}\left(\frac{\delta \partial^{\mu} \psi}{1-2 g \delta}\right)
$$

and

$$
\partial_{\mu} \partial^{\mu} \delta-\frac{2}{r^{2}} \delta=g \frac{\partial_{\mu} \psi \partial^{\mu} \psi}{1-2 g \delta}-g \frac{\partial_{\mu} \delta \partial^{\mu} \delta}{1-2 g \delta}-\frac{4 g}{r^{2}} \delta^{2}
$$

and the energy (2.13) is

$$
E=8 \pi \int_{0}^{\infty} d r\left[\frac{1}{2(1-2 g \delta)}\left\{\left(\partial_{t} \delta\right)^{2}+\left(\partial_{r} \delta\right)^{2}+\left(\partial_{t} \psi\right)^{2}+\left(\partial_{r} \psi\right)^{2}\right\}+\frac{\psi^{2}}{r^{2}}+\frac{\delta^{2}}{r^{2}}\right]
$$

We will show how to solve 2.15) in a perturbative expansion in $g$ after we show that these equations generically describe imploding shells in the far past which turn into outgoing shells in the far future. 


\section{LINEARIZATION IN THE FAR PAST AND FAR FUTURE}

In the introduction we pointed out that in the spherical ansatz a typical solution to the equations of motion describes an imploding shell, moving close to the speed of light, at very early times. To see how this comes about consider equations (2.15) and imagine that at some early time $T<0, \delta$ and $\psi$ are both pulses of width $\Delta$ centered at $r$ near $|T|$ with $\Delta \ll|T|$. By a pulse we mean a function which is very close to zero except in a region whose size is $\Delta$. Accordingly, space and time derivatives of $\psi$ and $\delta$ are pulses with amplitude of order $\psi / \Delta$ and $\delta / \Delta$. For $r \simeq|T| \gg \Delta$ we can now neglect the $1 / r^{2}$ terms in (2.15). We then see that if $\psi(r, t)$ and $\delta(r, t)$ depend only on $r+t$, that is, $\psi(r, t)=\psi_{p}(r+t)$ and $\delta(r, t)=\delta_{p}(r+t)$ then equations (2.15) are satisfied. If $\psi_{p}(u)$ and $\delta_{p}(u)$ are close to zero except for $u$ in a region of size $\Delta$ around $u=0$, then the solution describes an incoming shell of width $\Delta$ moving undistorted along $r=-t$. This description remains valid for all $t \ll-\Delta$.

Although equations (2.15) with the $1 / r^{2}$ terms neglected have solutions $\psi(r, t)=\psi_{p}(r+t)$ and $\delta(r, t)=\delta_{p}(r+t)$, these equations are not linear. We are for example not assuming that $\delta$ and $\psi$ are small. There is however a sense in which we can say that the theory linearizes. Consider the field strength $F_{\mu \nu}$ in the $3+1$ dimensional theory given by (1.3). We now argue that there is a choice of gauge such that the commutator term, $\left[A_{\mu}, A_{\nu}\right]$, can be neglected relative to the linear term, $\partial_{\mu} A_{\nu}-\partial_{\nu} A_{\mu}$, for all $t$ much less than $-\Delta$. Clearly this statement requires a gauge choice since the size of $A_{\mu}$ and its derivatives are gauge dependent. Nonetheless the statement that there exists a gauge with this property is a gauge invariant characterization of solutions in the spherical ansatz.

Work in the $A_{0}=0$ gauge which in the reduced theory is equivalent to the $a_{0}=0$ gauge. Using (2.11) and (2.14) we then have

$$
\partial_{r} \psi(r, t)=(2 g \delta(r, t)-1) \partial_{t} \varphi(r, t)
$$

or 


$$
\varphi(r, t)=-\int_{-\infty}^{t} d t^{\prime} \frac{\partial_{r} \psi\left(r, t^{\prime}\right)}{1-2 g \delta\left(r, t^{\prime}\right)}+\varphi_{0}(r)
$$

where $\varphi_{0}(r)$ is an arbitrary function of $r$. Now for $t \ll-\Delta$, using $\psi_{p}(r+t)$ and $\delta_{p}(r+t)$ for $\psi$ and $\delta$, we have

$$
\varphi(r, t)=-\int_{-\infty}^{r+t} d u \frac{\partial_{u} \psi_{p}(u)}{1-2 g \delta_{p}(u)}+\varphi_{0}(r)
$$

Equations (2.11) and (2.14) also give, for all time,

$$
a_{1}(r, t)=\partial_{r} \varphi(r, t)+\frac{\partial_{t} \psi(r, t)}{1-2 g \delta(r, t)}
$$

which for $t \ll-\Delta$, upon using (3.3), becomes

$$
a_{1}(r, t)=\partial_{r} \varphi_{0}(r)
$$

In the $a_{0}=0$ gauge we are still free to make a time independent gauge transformation which we do to set

$$
a_{1}(r, t)=0
$$

for $t \ll-\Delta$. What we have shown is that in the $a_{0}=0$ gauge if we set $a_{1}=0$ at one early time then we have $a_{1}=0$ at all early times.

If we now turn to (2.1) we see that in the gauge we have chosen, at early times, the dominant pieces in $A_{i}$ go like $\alpha / r$ and $\gamma / r$. Since the gauge has been specified, $\alpha$ and $\gamma$ are determined by $\delta$ and $\psi$ so, for example, derivatives of $\alpha$ and $\gamma$ are of order $\alpha / \Delta$ and $\gamma / \Delta$. This means that $\partial_{\mu} A_{i}$ is of order $\alpha /(r \Delta)$ and $\gamma /(r \Delta)$ whereas $A_{i} A_{j}$ is of order $\alpha^{2} / r^{2}, \alpha \gamma / r^{2}$ and $\gamma^{2} / r^{2}$. For $\Delta \ll r$ the commutator term in $F_{\mu \nu}$ can be neglected relative to the linear term. (In fact $F_{\mu \nu}$ goes like $1 /(r \Delta)$ so the total energy in the shell scales as $1 / \Delta$.) We also see that the energy is, at early times, quadratic in the field $A_{i}$ which is a signature of a linear theory.

We are particularly interested in solutions which in the far past describe free particles. Thus we want the gauge field $A_{\mu}$, in the gauge in which the solution linearizes in the far 
past, to be independent of $g$ in the far past. In this case $F_{\mu \nu}$ is also independent of $g$ since $F_{\mu \nu} \simeq \partial_{\mu} A_{\nu}-\partial_{\nu} A_{\mu}$. Then, for example, the energy which depends on $F_{\mu \nu}^{2}$ is independent of $g$.

In the gauge in which the solutions linearize, $a_{0}=0$ and $a_{1}=0$ for $t \ll-\Delta$. For $A_{\mu}$ in this regime to be independent of $g$, we require that $\alpha$ and $\gamma$ be independent of $g$ (see (2.1)). Now from (2.5), (2.8) and (2.14), we have

$$
\delta=\gamma-\frac{1}{2} g\left(\alpha^{2}+\gamma^{2}\right)
$$

Using (2.5), (2.8), (2.14) and (3.1), we also have

$$
\partial_{r} \psi=-\partial_{t} \alpha+g\left(\gamma \partial_{t} \alpha-\alpha \partial_{t} \gamma\right)
$$

This means that if in the far past $\alpha$ and $\gamma$ are independent of $g$, then the gauge invariant variables $\psi$ and $\delta$ have pieces which are zeroth and first order in $g$. If $\alpha(r, t)$ and $\gamma(r, t)$ for $t \ll-\Delta$ are described by pulses $\alpha(r, t)=\alpha_{p}(r+t)$ and $\gamma(r, t)=\gamma_{p}(r+t)$ then

$$
\delta_{p}(u)=\gamma_{p}(u)-\frac{1}{2} g\left(\alpha_{p}^{2}(u)+\gamma_{p}^{2}(u)\right)
$$

and

$$
\partial_{u} \psi_{p}(u)=-\partial_{u} \alpha_{p}(u)+g\left(\gamma_{p}(u) \partial_{u} \alpha_{p}(u)-\alpha_{p}(u) \partial_{u} \gamma_{p}(u)\right)
$$

Note that if we assume this form for $\delta_{p}(u)$ and $\psi_{p}(u)$, then the energy evaluated using (2.16), neglecting the $1 / r^{2}$ terms, is indeed independent of $g$.

We have shown that an incoming pulse moving undistorted at the speed of light, $\psi(r, t)=$ $\psi_{p}(r+t)$ and $\delta(r, t)=\delta_{p}(r+t)$, solves the equation of motion (2.15), as long as the pulse is located at a radius much larger than the width of the pulse. Similarly an outgoing pulse, $\psi(r, t)=\psi_{f}(t-r)$ and $\delta(r, t)=\delta_{f}(t-r)$ solves the equations of motion under the same conditions. A general scattering solution consists of an incoming pulse in the far past which in the far future is an outgoing pulse. The future pulse profiles, $\psi_{f}$ and $\delta_{f}$ will not, in general, coincide with the past pulse profiles $\psi_{p}$ and $\delta_{p}$. In fact a full understanding of the solutions to (2.15) would allow us to determine $\psi_{f}$ and $\delta_{f}$ given arbitrary $\psi_{p}$ and $\delta_{p}$. 


\section{THE PERTURBATIVE EXPANSION}

In this section we solve equations (2.15) perturbatively in $g$ in an in-field formalism where we specify the form of the solution in the far past. If we set $g$ equal to zero, equations (2.15a) and (2.15b) have the same form:

$$
\left(-\partial_{t}^{2}+\partial_{r}^{2}-\frac{2}{r^{2}}\right) \psi_{0}=0
$$

and

$$
\left(-\partial_{t}^{2}+\partial_{r}^{2}-\frac{2}{r^{2}}\right) \delta_{0}=0
$$

It is convenient to switch to light cone coordinates defined as

$$
\begin{aligned}
& u=t+r \\
& v=t-r,
\end{aligned}
$$

in which $d s^{2}=-d t^{2}+d r^{2}=-d u d v$ and $\partial_{\mu} \partial^{\mu}=-\partial_{t}^{2}+\partial_{r}^{2}=-4 \partial_{u} \partial_{v}$. Finite energy requires the solutions to vanish at $r=(u-v) / 2=0$. The general finite energy solution to the $g=0$ homogeneous equations $(4.1)$ is

$$
\psi_{0}(u, v)=G^{\prime}(u)+G^{\prime}(v)-\frac{2}{u-v}[G(u)-G(v)]
$$

and

$$
\delta_{0}(u, v)=H^{\prime}(u)+H^{\prime}(v)-\frac{2}{u-v}[H(u)-H(v)]
$$

where $G(x)$ and $H(x)$ are two arbitrary functions which approach constants as $x$ goes to $\pm \infty$. If $G^{\prime}(x)$ and $H^{\prime}(x)$ are both nonzero only in a region of size $\Delta$ containing $x=0$, then the solution represents an energy shell of width $\Delta$ moving along $r=-t$ at early times $(t \ll-\Delta)$ and along $r=t$ at late times $(t \gg \Delta)$.

We now expand the fields $\psi$ and $\delta$ in the coupling $g$ :

$$
\begin{gathered}
\psi=\psi_{0}+g \psi_{1}+g^{2} \psi_{2}+\ldots, \\
\delta=\delta_{0}+g \delta_{1}+g^{2} \delta_{2}+\ldots
\end{gathered}
$$


and substitute into the equations of motion (2.15). Expanding the equations in $g$ gives

$$
\begin{gathered}
{\left[\partial_{u} \partial_{v}+\frac{2}{(u-v)^{2}}\right] \psi_{1}=I_{1}^{\psi},} \\
{\left[\partial_{u} \partial_{v}+\frac{2}{(u-v)^{2}}\right] \delta_{1}=I_{1}^{\delta},} \\
\vdots \\
{\left[\partial_{u} \partial_{v}+\frac{2}{(u-v)^{2}}\right] \psi_{n}=I_{n}^{\psi},} \\
{\left[\partial_{u} \partial_{v}+\frac{2}{(u-v)^{2}}\right] \delta_{n}=I_{n}^{\delta},}
\end{gathered}
$$

where $I_{n}^{\psi}$ and $I_{n}^{\delta}$ both depend only on $\psi_{0}, \ldots \psi_{n-1}$ and $\delta_{0}, \ldots \delta_{n-1}$. For example

$$
\begin{aligned}
I_{1}^{\psi} & =\frac{1}{2} \partial_{\mu}\left(\delta_{0} \partial^{\mu} \psi_{0}\right) \\
I_{1}^{\delta} & =\frac{1}{4} \partial_{\mu} \delta_{0} \partial^{\mu} \delta_{0}-\frac{1}{4} \partial_{\mu} \psi_{0} \partial^{\mu} \psi_{0}+\frac{4 \delta_{0}^{2}}{(u-v)^{2}} \\
I_{2}^{\psi} & =\frac{1}{2} \partial_{\mu}\left(\delta_{1} \partial^{\mu} \psi_{0}+\delta_{0} \partial^{\mu} \psi_{1}+2 \delta_{0}^{2} \partial^{\mu} \psi_{0}\right) \\
I_{2}^{\delta} & =\frac{1}{2} \delta_{0} \partial_{\mu} \delta_{0} \partial^{\mu} \delta_{0}-\frac{1}{2} \delta_{0} \partial_{\mu} \psi_{0} \partial^{\mu} \psi_{0}-\frac{1}{2} \partial_{\mu} \psi_{1} \partial^{\mu} \psi_{0}+\frac{1}{2} \partial_{\mu} \delta_{1} \partial^{\mu} \delta_{0}+\frac{8 \delta_{1} \delta_{0}}{(u-v)^{2}}
\end{aligned}
$$

Given the sources $I_{n}^{\psi}$ and $I_{n}^{\delta}$, equations (4.5) do not uniquely specify $\psi_{n}$ and $\delta_{n}$ because we can add homogeneous solutions of the form (4.3a) and (4.3b) to $\psi_{n}$ and $\delta_{n}$ and still satisfy the equations. We will first solve (4.5) with the requirement that the solutions vanish in the far past, and will later use the freedom to add homogeneous solutions to find solutions which describe $g$-independent $\alpha$ and $\gamma$ pulses in the far past. Consider the equation

$$
\left[\partial_{u} \partial_{v}+\frac{2}{(u-v)^{2}}\right] \Phi(u, v)=I(u, v)
$$

where the source $I \rightarrow 0$ as $v \rightarrow-\infty$, which we solve requiring that the solution $\Phi \rightarrow 0$ as $v \rightarrow-\infty$, and also that $\Phi$ vanishes at $r=(u-v) / 2=0$. The retarded Green's function solution is

$$
\Phi(u, v)=\int_{v}^{u} d u^{\prime} \int_{-\infty}^{v} d v^{\prime} F\left(u, v ; u^{\prime}, v^{\prime}\right) I\left(u^{\prime}, v^{\prime}\right)
$$


where

$$
F\left(u, v ; u^{\prime}, v^{\prime}\right)=1-\frac{2\left(u-u^{\prime}\right)\left(v-v^{\prime}\right)}{(u-v)\left(u^{\prime}-v^{\prime}\right)}
$$

(Note that $F\left(u, v ; u^{\prime}, v^{\prime}\right)$ satisfies $(4.7)$ with $I=0$. )

We can use (4.8) to get explicit formulas for $\psi_{1}$ and $\delta_{1}$ given the sources $I_{1}^{\psi}$ and $I_{1}^{\delta}$ of (4.6a) and (4.6b) along with $\psi_{0}$ and $\delta_{0}$ expressed in terms of the arbitrary functions $G(x)$ and $H(x)$ of $(4.3 \mathrm{a})$ and $(4.3 \mathrm{~b})$. The result is

$$
\begin{aligned}
\psi_{1}(u, v)= & -G^{\prime}(u) H^{\prime}(v)-G^{\prime}(v) H^{\prime}(u)-\frac{6}{(u-v)^{2}}[G(u)-G(v)][H(u)-H(v)] \\
& +\frac{2}{u-v}\left[G^{\prime}(u)+G^{\prime}(v)\right][H(u)-H(v)] \\
& +\frac{2}{u-v}\left[H^{\prime}(u)+H^{\prime}(v)\right][G(u)-G(v)]
\end{aligned}
$$

and

$$
\begin{aligned}
\delta_{1}(u, v)= & G^{\prime}(u) G^{\prime}(v)+\frac{[G(u)-G(v)]^{2}}{(u-v)^{2}}-\frac{2}{u-v}\left[G^{\prime}(u)+G^{\prime}(v)\right][G(u)-G(v)] \\
& +\frac{2}{u-v} \int_{v}^{u} d x G^{\prime}(x)^{2}-H^{\prime}(u) H^{\prime}(v)-\frac{5}{(u-v)^{2}}[H(u)-H(v)]^{2} \\
& +\frac{2}{u-v}\left[H^{\prime}(u)+H^{\prime}(v)\right][H(u)-H(v)]+\frac{2}{u-v} \int_{v}^{u} d x H^{\prime}(x)^{2} .
\end{aligned}
$$

Using the fact that $G^{\prime}(x)$ and $H^{\prime}(x)$ are only nonvanishing near $x=0$ we see that $\psi_{1}$ and $\delta_{1}$ do in fact go to zero as $v \rightarrow-\infty$. We are still free to add homogeneous pieces to $\psi_{1}$ and $\delta_{1}$ if we so choose. Indeed we will need to use this freedom since equations (3.9) and the requirement that $\alpha$ and $\gamma$ are $g$-independent in the far past imply that $\psi_{0}, \psi_{1}, \delta_{0}$, and $\delta_{1}$ are in general nonzero as $v \rightarrow-\infty$. Given $\psi_{1}$ and $\delta_{1}$ we can evaluate $I_{2}^{\psi}$ and $I_{2}^{\delta}$ in $(4.6 \mathrm{c}$ ) and (4.6d) and then obtain $\psi_{2}$ and $\delta_{2}$ using (4.8). Continuing along these lines we can, in principle, get $\psi$ and $\delta$ to any order in $g$. Of course in practice the calculations get more complicated with each order of perturbation theory. 


\section{TOPOLOGICAL CHARGE}

In the spherical ansatz, using the equations of motion, the local topological charge (1.1) can be written [0] as

$$
Q(R)=\frac{g}{2 \pi} \int_{-\infty}^{\infty} d t \int_{0}^{R} d r\left(-\partial_{t}^{2}+\partial_{r}^{2}-\frac{2}{r^{2}}\right) \psi
$$

where $\psi(r, t)$ is a solution to (2.15). Since the integrand in (1.1) is a total divergence we can also write $Q(R)$ as a surface integral. Using (2.15a) we have that

$$
Q(R)=\frac{-g^{2}}{\pi} \int_{-\infty}^{\infty} d t \int_{0}^{R} d r \partial_{\mu}\left\{\frac{\delta \partial^{\mu} \psi}{1-2 g \delta}\right\}
$$

or

$$
Q(R)=\left.\frac{-g^{2}}{\pi} \int_{-\infty}^{\infty} d t \frac{\delta \partial_{r} \psi}{1-2 g \delta}\right|_{r=R}
$$

since the contributions from the $t= \pm \infty$ surfaces and the $r=0$ surface vanish.

We are interested in $R$ much larger than any length scale associated with the solution. At this large value of $R$, the integral in (5.3) is only nonzero when the incoming pulse passes $R$ at $t \simeq-R$ and when the outgoing pulse passes $R$ at $t \simeq R$. For $t \simeq-R$ we have $\psi(R, t)=\psi_{p}(R+t)+\mathcal{O}(1 / R)$ and $\delta(R, t)=\delta_{p}(R+t)+\mathcal{O}(1 / R)$ while for $t \simeq R$ we have $\psi(R, t)=\psi_{f}(t-R)+\mathcal{O}(1 / R)$ and $\delta(R, t)=\delta_{f}(t-R)+\mathcal{O}(1 / R)$. Here $\psi_{p}(u)=\psi(u,-\infty)$ and $\delta_{p}(u)=\delta(u,-\infty)$ are the incoming pulse functions described in section III and $\psi_{f}(v)=$ $\psi(\infty, v)$ and $\delta_{f}(v)=\delta(\infty, v)$ are the associated future pulse functions. Substituting into (5.3) and taking the limit as $R$ goes to infinity gives

$$
Q=\frac{g^{2}}{\pi} \int_{-\infty}^{\infty} d v\left\{\frac{\delta_{f}(v) \partial_{v} \psi_{f}(v)}{1-2 g \delta_{f}(v)}\right\}-\frac{g^{2}}{\pi} \int_{-\infty}^{\infty} d u\left\{\frac{\delta_{p}(v) \partial_{u} \psi_{p}(u)}{1-2 g \delta_{p}(u)}\right\}
$$

Note that topological charge develops only to the extent that the outgoing profiles differ from the incoming profiles.

We can develop (5.4) perturbatively in $g$ if we use the perturbative expansion for the solutions $\psi(u, v)$ and $\delta(u, v)$. To zeroth order in $g, \psi(u, v)$ and $\delta(u, v)$ are given by (4.3a) 
and (4.3b) so we see that to this order, the future and past profile functions agree, that is, $\psi_{f 0}(x)=\psi_{p 0}(x)$ and $\delta_{f 0}(x)=\delta_{p 0}(x)$. This means that the topological charge vanishes to second order in $g$.

If we look at (1.2) along with (1.3) and (1.4) we see that the second order topological charge, outside the spherical ansatz, can be written as

$$
Q^{[2]}=\frac{g^{2}}{16 \pi^{2}} \sum_{a=1}^{3} \int d^{4} x \epsilon^{\mu \nu \alpha \beta} \partial_{\mu} A_{\nu}^{a} \partial_{\alpha} A_{\beta}^{a}
$$

where

$$
\partial^{\mu}\left(\partial_{\mu} A_{\nu}^{a}-\partial_{\nu} A_{\mu}^{a}\right)=0
$$

Thus the second order non-Abelian topological charge is equivalent to the topological charge in an Abelian theory. In appendix A we show, without restricting to the spherical ansatz, that for localized energy pulses which solve Maxwell's equations, the topological charge vanishes.

To get the third order topological charge we need $\psi_{1}(u, v)$ and $\delta_{1}(u, v)$ whose general form is given by (4.10) plus possible homogeneous pieces. With $\psi_{1}$ and $\delta_{1}$ given by (4.10), we see that both go to zero for large positive $u$ and for large negative $v$ so that $\psi_{f 1}=\psi_{p 1}=$ $\delta_{f 1}=\delta_{p 1}=0$. Any homogeneous piece in $\psi_{1}$ and $\delta_{1}$ will make cancelling contributions to (5.4) so we have that to order $g^{3}$ the topological charge vanishes within the spherical ansatz.

The fourth order topological charge is more difficult to evaluate. For arbitrary initial profiles we would need to find $\psi_{2}$ and $\delta_{2}$ which entails evaluating (4.8) with the sources (4.60) and (4.6d) where $\psi_{0}$ and $\delta_{0}$ are given by (4.3) and $\psi_{1}$ and $\delta_{1}$ are given by (4.10) plus possible homogeneous terms. We have done this only in examples. For the examples considered we find that $\psi_{f 2}$ and $\delta_{f 2}$ are non-vanishing even though $\psi_{p 2}=\delta_{p 2}=0$. Nonetheless, in every case the fourth order topological charge vanishes because of cancellations in the first term in (5.4). Our goal is not to prove that the fourth order topological charge is zero for arbitrary initial profiles. Our goal is to show that there are initial profiles for which $Q$ is of order $g^{n}$ for some finite $n$. Therefore, onward to the $g^{5}$ term. 
We have evaluated the fifth order topological charge for several initial profiles. In every case, we have found a nonzero result. In principle, once the initial conditions are specified the calculation of the fifth order topological charge follows from (5.4) and the results of section IV. In practice, at this order the calculations are rather involved and we relegate the details to an appendix. Here, we will describe the initial conditions we use, and quote the resulting $Q^{[5]}$ for two examples.

Initial conditions are specified by the functions $\gamma_{p}(u)$ and $\alpha_{p}(u)$ of equations (3.9) which we require to be $g$-independent. We make the choice $\gamma_{p}(u)=0$ because it simplifies the calculation considerably. From (3.9) and (4.3), this choice implies $G^{\prime}(x)=-\alpha_{p}(x)$ and $H(x)=0$. Because $H(x)=0$, equation (4.10a) gives $\psi_{1}(u, v)=0$, which agrees with the order $g$ term of (3.9b). In general, the equations of motion (2.15) are invariant under the transformation $g \rightarrow-g$ and $\delta \rightarrow-\delta$ with $\psi$ fixed. For $\gamma_{p}=0$, the initial conditions (3.9) are invariant also. This means that for $\gamma_{p}=0$, the expansion in $g$ of $\delta$ can contain only odd powers of $g$, while that of $\psi$ can contain only even powers of $g$. The order $g$ term of (3.9a) requires that $\delta_{p 1}(u)=-\frac{1}{2} G^{\prime}(u)^{2}$. Therefore, we must add the homogeneous term

$$
\delta_{1}^{\text {hom }}(u, v)=-\frac{1}{2}\left[G^{\prime}(u)^{2}+G^{\prime}(v)^{2}-\frac{2}{u-v} \int_{v}^{u} d x G^{\prime}(x)^{2}\right]
$$

to $\delta_{1}$ of $(4.10 \mathrm{~b})$ to obtain

$$
\begin{aligned}
\delta_{1}(u, v)= & -\frac{1}{2}\left[G^{\prime}(u)-G^{\prime}(v)\right]^{2}+\frac{[G(u)-G(v)]^{2}}{(u-v)^{2}} \\
& -\frac{2}{u-v}\left[G^{\prime}(u)+G^{\prime}(v)\right][G(u)-G(v)]+\frac{3}{u-v} \int_{v}^{u} d x G^{\prime}(x)^{2} .
\end{aligned}
$$

Thus, choosing $\gamma_{p}=0$ means that $\delta_{0}$ and $\psi_{1}$ are zero for all $u$ and $v$, while $\psi_{0}(u, v)$ and $\delta_{1}(u, v)$ are given in terms of the single function $G(x)$ by equations (4.3a) and (5.8).

In appendix $\mathrm{B}$, we show that for $\gamma_{p}=0$, the fifth order term in the topological charge (5.4) can be written as the double integral

$$
\begin{aligned}
Q^{[5]}=\frac{g^{5}}{\pi} \int_{-\infty}^{\infty} d u d v\{ & \frac{1}{2}\left[\frac{1}{2} \partial_{\mu} \psi_{0} \partial^{\mu} \delta_{1}+\frac{4 \psi_{0} \delta_{1}}{(u-v)^{2}}\right]\left[2 G^{\prime}(u) G^{\prime}(v)+\frac{6}{(u-v)^{2}}[G(u)-G(v)]^{2}\right. \\
& \left.-\frac{4}{(u-v)^{2}}\left[G^{\prime}(u)+G^{\prime}(v)\right][G(u)-G(v)]\right] \\
& \left.-\frac{3}{2} \frac{\psi_{0} \delta_{1}}{(u-v)^{2}}-\frac{\psi_{0}^{3} \delta_{1}}{(u-v)^{2}}-\frac{1}{8} \frac{\psi_{0}^{5}}{(u-v)^{2}}\right\} .
\end{aligned}
$$


We have evaluated equation (5.9) analytically for several functions $G(x)$. Here, we present the results for two examples. For

$$
G(x)=-\frac{x}{\left(1+x^{2}\right)}
$$

we find

$$
Q^{[5]}=-\frac{15 \pi^{2}}{1024} \frac{g^{5}}{\pi}
$$

For

$$
G^{\prime}(x)= \begin{cases}0 ; & x \leq-1 \\ \left(1-x^{2}\right)^{2} ; & -1 \leq x \leq 1 \\ 0 ; & 1 \leq x\end{cases}
$$

we find

$$
Q^{[5]}=\frac{1336082432}{11402015625} \frac{g^{5}}{\pi}
$$

We have demonstrated that spherical solutions to the Minkowski space classical equations of motion for pure $S U(2)$ which propagate freely in the far past can have nonzero topological charge. The power series expansion of $Q$ in $g$ can be nonzero at finite order - in the examples we have presented, at order $g^{5}$.

\section{FERMION PRODUCTION IN A BACKGROUND FIELD WITH FRACTIONAL TOPOLOGICAL CHARGE}

The solutions which we consider in this paper have the property that the topological charge inside a sphere of radius $R, Q(R)$ given by (1.1), approaches a fixed value as $R$ gets large and the limiting value, $Q=\lim _{R \rightarrow \infty} Q(R)$, need not be an integer. In fact we have shown that $Q$ can be developed as a power series in $g$ so that $Q$ can be made small by making $g$ small. Suppose that we have a quantized left-handed fermi field, $\hat{\Psi}$, coupled to this classical non-Abelian background. Without introducing a Higgs field, we cannot give the fermion a gauge invariant mass, so we take the fermion to be massless. Due to the anomaly, the fermion number current $\hat{J}^{\mu}=: \hat{\bar{\Psi}} \gamma^{\mu} \hat{\Psi}:$ is not conserved, that is, 


$$
\partial_{\mu} \hat{J}^{\mu}=\frac{g^{2}}{32 \pi^{2}} \epsilon^{\mu \nu \alpha \beta} \operatorname{Tr}\left(F_{\mu \nu} F_{\alpha \beta}\right)
$$

The question arises of how many fermions are produced in the presence of a background field of the kind we have described.

Define the fermion number operator, inside the sphere of radius $R$, as

$$
\hat{N}(R, t)=\int_{0}^{R} r^{2} d r \int d \cos \theta d \phi \hat{J}^{0}(r, \theta, \phi, t)
$$

and the flux operator, through the two-sphere of radius $R$, as

$$
\hat{F}(R, t)=\int d \cos \theta d \phi R^{2} \hat{J}^{r}(R, \theta, \phi, t) .
$$

(We will for the moment ignore the fact that these are not well defined operators and return to this point later.) Now, integrating (6.1) from $t=-T_{0}$ to $t=T_{0}$ and over the inside of the sphere of radius $R$, we obtain

$$
\hat{N}\left(R, T_{0}\right)-\hat{N}\left(R,-T_{0}\right)+\int_{-T_{0}}^{T_{0}} d t \hat{F}(R, t)=\frac{g^{2}}{32 \pi^{2}} \epsilon^{\mu \nu \alpha \beta} \int_{-T_{0}}^{T_{0}} d t \int_{|\vec{x}|<R} d^{3} x \operatorname{Tr}\left(F_{\mu \nu} F_{\alpha \beta}\right) .
$$

We are interested in background shells which at early times are moving along $r=-t$, enter the sphere of radius $R$ at $t \simeq-R$, leave at $t \simeq R$, and then continue to move outward along $r=t$. If we take $T_{0}$ much larger than $R$, then the right hand side of (6.3) is well approximated by $Q(R)$ of (5.1) and we can write

$$
\hat{N}\left(R, T_{0}\right)-\hat{N}\left(R,-T_{0}\right)+\int_{-T_{0}}^{T_{0}} d t \hat{F}(R, t)=Q(R) .
$$

We are interested in tracing the evolution of a normalized fermion state $|s\rangle$ in the background of a shell which enters and leaves the sphere of radius $R$. Pick the state $|s\rangle$ such that at $t=-T_{0}$ there are no fermions inside the sphere, which implies

$$
\left\langle s\left|\hat{N}\left(R,-T_{0}\right)\right| s\right\rangle=0 .
$$

The non-Abelian shell may have fermions riding along with it which enter and leave the sphere of radius $R$ making $\langle s|\hat{F}(R, t)| s\rangle$ nonzero for $t \simeq-R$ and $t \simeq R$. Any fermions anomalously produced within the sphere will also contribute to $\langle s|\hat{F}(R, t)| s\rangle$ when they 
leave the sphere. For $t \gg R$, the energy shell is far outside the sphere and the background field is once again pure gauge within the sphere. Therefore, at late times fermions inside the sphere are free, and if we wait long enough they will leave the sphere. Thus, for $T_{0}$ sufficiently large, we have

$$
\left\langle s\left|\hat{N}\left(R, T_{0}\right)\right| s\right\rangle=0 .
$$

Taking the expectation of (6.4) in the state $|s\rangle$, we obtain

$$
\int_{-T_{0}}^{T_{0}} d t\langle s|\hat{F}(R, t)| s\rangle=Q(R) .
$$

We interpret (6.7) as follows. Imagine a spherical detector corresponding to the operator (6.2b), which at any time $t$ measures the flux of fermions passing out through the two sphere of radius $R$. (Fermions going in make a negative contribution, as do anti-fermions going out.) The detector is in continuous operation from $t=-T_{0}$ to $t=T_{0}$ and therefore measures the total fermion number passing through the two sphere. For a given background field, we cannot predict the outcome of an individual measurement of the total fermion number passing through the two sphere. However, if we repeat the measurement many times with the same background field and the same state $|s\rangle$, then the average of the observations will be $Q(R)$.

Although we believe that the spirit of the argument just given is correct, it is specious because the operators $\hat{N}(R, t)$ and $\hat{F}(R, t)$ have infinite vacuum fluctuations, that is,

$$
\left\langle 0\left|\hat{N}^{2}(R, t)\right| 0\right\rangle=\infty
$$

and

$$
\left\langle 0\left|\hat{F}^{2}(R, t)\right| 0\right\rangle=\infty .
$$

Therefore, these operators also have infinite fluctuations in any state in the Fock space; in particular $\left\langle s\left|\hat{N}^{2}(R, t)\right| s\right\rangle$ is infinite. Thus, we cannot sensibly discuss the measurement of these operators. 
The infinite vacuum fluctuations arise because of the sharp cutoffs in the $r$ integration at $r=R$ and in the $t$ integration at $t= \pm T_{0}$ in expressions (6.2a), (6.2b), and (6.3). This can be remedied by introducing a smoothing function. Let

$$
f(r, t)= \begin{cases}1, & \text { if } 0 \leq r \leq R \text { and }|t| \leq T_{0} \\ 0, & \text { if } r \geq R_{D} \text { or }|t| \geq T_{D}\end{cases}
$$

and let $f(r, t)$ fall smoothly from 1 to 0 as $r$ varies from $R$ to $R_{D}$ and as $|t|$ varies from $T_{0}$ to $T_{D}$. Define the smoothed operators

$$
\hat{N}_{f}=-\int_{-\infty}^{\infty} d t \int_{0}^{\infty} r^{2} d r \int d \cos \theta d \phi \frac{\partial f(r, t)}{\partial t} \hat{J}^{0}(r, \theta, \phi, t)
$$

and

$$
\hat{F}_{f}=-\int_{-\infty}^{\infty} d t \int_{0}^{\infty} r^{2} d r \int d \cos \theta d \phi \frac{\partial f(r, t)}{\partial r} \hat{J}^{r}(r, \theta, \phi, t)
$$

Because $\partial f(r, t) / \partial t$ is only nonzero for $T_{0} \leq|t| \leq T_{D}$, it is also convenient to define

$$
\hat{N}_{f}^{\text {early }}=\int_{-T_{D}}^{-T_{0}} d t \int_{0}^{\infty} r^{2} d r \int d \cos \theta d \phi \frac{\partial f(r, t)}{\partial t} \hat{J}^{0}(r, \theta, \phi, t)
$$

and

$$
\hat{N}_{f}^{\text {late }}=-\int_{T_{0}}^{T_{D}} d t \int_{0}^{\infty} r^{2} d r \int d \cos \theta d \phi \frac{\partial f(r, t)}{\partial t} \hat{J}^{0}(r, \theta, \phi, t)
$$

where $\hat{N}_{f}^{\text {late }}-\hat{N}_{f}^{\text {early }}=\hat{N}_{f}$. Note that the eigenvalues of $\hat{N}_{f}, \hat{F}_{f}, \hat{N}_{f}^{\text {early }}$, and $\hat{N}_{f}^{\text {late }}$, are not integers. A short calculation shows that the vacuum fluctuations of the smoothed currents are given by

$$
\int d^{4} x \int d^{4} y f(x) f(y)\left\langle 0\left|\hat{J}^{\mu}(x) \hat{J}^{\nu}(y)\right| 0\right\rangle=\frac{1}{3} \int \frac{d^{4} k}{(2 \pi)^{5}} \theta\left(k^{0}\right) \theta\left(k^{2}\right)|\tilde{f}(k)|^{2}\left(k^{\mu} k^{\nu}-g^{\mu \nu} k^{2}\right)
$$

where $\tilde{f}$ is the fourier transform of $f$, and the currents are understood to be normal ordered. From (6.12), we see that by a judicious choice of $f(r, t)$, the vacuum fluctuations can be made arbitrarily small. For the massless fermion case which we are considering this requires $R_{D}$ to be much larger than $R$ and $T_{D}$ to be much larger than $T_{0}$. We also require that 
$T_{0} \gg R_{D}$ so that during the measurements of $\hat{N}_{f}^{\text {early }}$ and $\hat{N}_{f}^{\text {late }}$ the energy shell is far outside the detector. To summarize the relations between the relevant length scales, we have $\Delta \ll R \ll R_{D} \ll T_{0} \ll T_{D}$ where again $\Delta$ is the length which characterizes the pulse.

If we multiply both sides of (6.1) by $f(r, t)$ and integrate, we get

$$
\hat{N}_{f}^{\text {late }}-\hat{N}_{f}^{\text {early }}+\hat{F}_{f}=\frac{g^{2}}{32 \pi^{2}} \epsilon^{\mu \nu \alpha \beta} \int_{-\infty}^{\infty} d t \int d^{3} x f(r, t) \operatorname{Tr}\left(F_{\mu \nu} F_{\alpha \beta}\right) .
$$

Using the equations of motion for the fields in the spherical ansatz, we can write the right hand side of (6.13) as

$$
\frac{g}{2 \pi} \int_{-\infty}^{\infty} d t \int_{0}^{\infty} d r f(r, t)\left(-\partial_{t}^{2}+\partial_{r}^{2}-\frac{2}{r^{2}}\right) \psi
$$

For $r \gg \Delta$, and in particular for $r \geq R$, the integrand of (6.14) is well approximated by $f(r, t)\left(-\partial_{t}^{2}+\partial_{r}^{2}\right) \psi$ which is near zero because wherever and whenever $\psi$ is nonzero at large $r$, it is given either by $\psi_{p}(t+r)$ or by $\psi_{f}(t-r)$ up to terms of order $1 / R$. Also, for $|t| \geq T_{0}$ the shell is outside $r=R$ and so at these early and late times $\psi$ is close to zero for $r \leq R$. Thus, the integrand of (6.14) is close to zero wherever and whenever $f(r, t) \neq 1$. Therefore, the expression (6.14) is $Q$ up to terms of order $1 / R$.

Taking the expectation of (6.13) in a state $|s\rangle$ which has

$$
\left\langle s\left|\hat{N}_{f}^{\text {early }}\right| s\right\rangle=0
$$

and neglecting terms of order $1 / R$, we obtain

$$
\left\langle s\left|\hat{F}_{f}\right| s\right\rangle=Q
$$

where again we argue that $\left\langle s\left|\hat{N}_{f}^{\text {late }}\right| s\right\rangle=0$ for sufficiently large $T_{0}$. As before, we interpret (6.16) in an expectation value sense. However in this case if the fluctuations of the flux operator are small enough, then each observation of the net number of fermions flowing through the detector corresponding to the smoothed operator (6.10b) will yield a number close to $Q$. In this sense, fermion number violation is seen with each observation. 


\section{CONCLUDING REMARKS AND OPEN QUESTIONS}

We have shown that spherical solutions to the classical equations of motion of $S U(2)$ gauge theory which propagate freely in the far past have nonzero topological charge. Furthermore, the topological charge of such solutions can be developed as a power series expansion in $g$. We presented examples for which $Q$ has a nonzero contribution at order $g^{5}$. In these examples $Q$ is nonzero for $g$-independent initial conditions. In particular, this suggests that in a corresponding quantum process, the number of particles in the initial state is $g$-independent, and need not be large at small $g$. We showed that if we couple quantized chiral fermions to classical gauge field solutions with nonzero $Q$, anomalous fermion number violation will occur. In a series of measurements using the operators of section VI with the same background gauge field and the same quantum mechanical fermion state, the average number of fermions anomalously produced is given by the topological charge $Q$. For the solutions we have discussed, the topological charge vanishes as a power of $g$. This suggests that anomalous fermion number violation in high energy scattering processes may occur at finite order in $g$, rather than being exponentially suppressed.

We cannot yet apply our results directly to scattering of $W$ bosons at energies much higher than the sphaleron energy because a number of important open questions remain to be considered. These questions arise because we have done a classical calculation in the

spherical ansatz for an $S U(2)$ gauge theory without a Higgs field. Let us consider each of these idealizations in turn.

First, in everything we have done, we have treated the gauge field classically, whereas we are ultimately interested in quantum scattering processes with a two-particle initial state. We need to relate our classical results to quantum mechanical scattering. One possible approach is to relate small amplitude classical solutions to quantum mechanical coherent states with small mean particle number. In this context, it is interesting to note that the $g$-expansion we have done is equivalent to an expansion in the amplitude of the initial pulse. Therefore, the topological charge at order $g^{5}$ is proportional to the fifth power of the 
amplitude of the initial pulse, and is not exponentially small for small amplitude pulses. This suggests that there may be scattering processes with few particles in the initial state in which $Q$ is not exponentially suppressed. Another way of addressing the problem might be to relate the lowest order term in a semi-classical expansion of the $S$-matrix for a quantum scattering process to the solutions of classical equations of motion for a specified initial configuration. The quantum lessons which our classical results are trying to teach us remain to be learned.

Second, we have worked within the spherical ansatz. This is a reasonable starting point since it makes the calculation tractable, and typically the lowest partial waves dominate scattering processes. An important open question here is that of stability. We have seen no evidence that the solutions we discuss are unstable to perturbations within the spherical ansatz. However, we have not checked their stability to non-spherical perturbations.

Finally, we have worked in $S U(2)$ gauge theory without a Higgs field. It remains to be determined how the inclusion of the Higgs field affects our results. It is possible that in scattering at very high energies the $W$ mass and sphaleron barrier are irrelevant and our results are a good approximation to results in the full theory. However, including the Higgs field changes the theory in qualitative ways. The theory is no longer scale invariant. The behavior we describe at early and late times — pulses propagating without distortion — will not occur once the Higgs field is included. Thus, while our results are likely applicable in the full theory in the limit of infinite energy scattering, it remains to be seen how they can be applied at high but finite energy.

\section{ACKNOWLEDGMENTS}

We would like to thank S. Coleman, J. Goldstone, T. Gould, S. Hsu, K. Johnson, V. Rubakov, and L. Yaffe for very helpful discussions. 


\section{APPENDIX A: ABELIAN TOPOLOGICAL CHARGE}

Here we show that for a localized solution to Maxwell's equations in empty space, the topological charge vanishes. We do not use the spherical ansatz. It is convenient to work with the gauge invariant electric and magnetic fields $\vec{E}(\vec{x}, t)$ and $\vec{B}(\vec{x}, t)$ which obey

$$
\begin{array}{ll}
\vec{\nabla} \cdot \vec{E}=0 & \vec{\nabla} \times \vec{E}=-\frac{\partial \vec{B}}{\partial t} \\
\vec{\nabla} \cdot \vec{B}=0 & \vec{\nabla} \times \vec{B}=\frac{\partial \vec{E}}{\partial t} .
\end{array}
$$

The most general solution can be expressed as

$$
\vec{E}(\vec{x}, t)=\int d^{3} k\left[\vec{\epsilon}(\vec{k}) \exp (i \omega t-i \vec{k} \cdot \vec{x})+\vec{\epsilon}^{*}(\vec{k}) \exp (-i \omega t+i \vec{k} \cdot \vec{x})\right]
$$

and

$$
\vec{B}(\vec{x}, t)=\int \frac{d^{3} k}{\omega}\left[\vec{k} \times \vec{\epsilon}(\vec{k}) \exp (i \omega t-i \vec{k} \cdot \vec{x})+\vec{k} \times \vec{\epsilon}^{*}(\vec{k}) \exp (-i \omega t+i \vec{k} \cdot \vec{x})\right]
$$

where $\omega=|\vec{k}|$ and $\vec{\epsilon}(\vec{k})$ is an arbitrary function of $\vec{k}$ subject to $\vec{k} \cdot \vec{\epsilon}(\vec{k})=0$.

Now the Abelian topological charge can be defined as

$$
Q^{\text {Abelian }}=\frac{1}{8 \pi^{2}} \int d^{4} x \vec{E} \cdot \vec{B}
$$

which in terms of $\vec{E}$ and $\vec{B}$ given above is seen to be

$$
Q^{\text {Abelian }}=-2 \pi^{2} \int_{0}^{\infty} \omega d \omega \int d \cos \theta d \varphi \delta(2 \omega)[\vec{\epsilon}(\vec{k}) \cdot(\vec{k} \times \vec{\epsilon}(-\vec{k}))+\text { c.c. }]
$$

The delta function in $\omega$ comes from the $\int d t$ in (A3). Because of this delta function, the integral will vanish unless $\vec{\epsilon}(\vec{k})$ goes like $1 / \omega$ as $\omega \rightarrow 0$. If we know $\vec{E}$ and $\vec{B}$ at one time, say $t=0$, we can determine $\vec{\epsilon}(\vec{k})$ :

$$
\vec{\epsilon}(\vec{k})=\frac{1}{2} \int \frac{d^{3} x}{(2 \pi)^{3}}\left[\vec{E}(\vec{x}, 0)-\frac{\vec{k}}{\omega} \times \vec{B}(\vec{x}, 0)\right] \exp i \vec{k} \cdot \vec{x} .
$$

As $\vec{k}$ goes to zero, $\vec{\epsilon}(\vec{k})$ will not blow up if

$$
\int d^{3} x \vec{E}(\vec{x}, 0)<\infty
$$


and

$$
\int d^{3} x \vec{B}(\vec{x}, 0)<\infty
$$

For localized pulses these conditions are satisfied and the Abelian topological charge vanishes. Note however that finite energy does not ensure (A6) so vanishing Abelian topological charge is not guaranteed under the most general circumstances.

\section{APPENDIX B: EVALUATING THE FIFTH ORDER TOPOLOGICAL CHARGE}

In this appendix we derive equation (5.9) for the fifth order topological charge for initial profiles for which $\gamma_{p}=0$ and $\alpha_{p}$ is independent of $g$. As was explained in the text, this implies that $\psi_{n}=0$ for $n$ odd and $\delta_{n}=0$ for $n$ even. We first need the third order contribution to the integrands of equation (5.4):

$$
\left(\frac{\delta \partial_{\mu} \psi}{1-2 g \delta}\right)^{[3]}=g^{3}\left(\delta_{3} \partial_{\mu} \psi_{0}+\delta_{1} \partial_{\mu} \psi_{2}+2 \delta_{1}^{2} \partial_{\mu} \psi_{0}\right) \text {. }
$$

From (5.8) we see that as $u \rightarrow \infty, \delta_{1} \rightarrow-(1 / 2) G^{\prime}(v)^{2}$ and as $v \rightarrow-\infty, \delta_{1} \rightarrow-(1 / 2) G^{\prime}(u)^{2}$. Therefore, the last term in (B1) makes cancelling contributions to the two terms of (5.4). Furthermore, there is no contribution from the first two terms of (B1) as $v \rightarrow-\infty$ since $\delta_{3}$ and $\psi_{2}$ vanish in this limit. Thus,

$$
Q^{[5]}=\left.\frac{g^{5}}{\pi} \int_{-\infty}^{\infty} d v\left(-\psi_{0} \partial_{v} \delta_{3}+\delta_{1}^{\mathrm{hom}} \partial_{v} \psi_{2}\right)\right|_{u=\infty}
$$

where the first term has been integrated by parts, and in the second term we have used the fact that at large $u$ we can replace $\delta_{1}$ by the homogeneous solution $\delta_{1}^{\text {hom }}$ given by (5.7).

These single integrals may be turned into double integrals using

$$
\left.\int_{-\infty}^{\infty} d v \eta \partial_{v} \Phi\right|_{u=\infty}=\int_{-\infty}^{\infty} d u \int_{-\infty}^{u} d v \eta I
$$

where $\Phi$, given by (4.8), is the solution to equation (4.7) with source $I$, and $\eta$ is any homogeneous solution of (4.7). This relation can be established by substituting (4.8) into the left hand side of (B3) and writing the homogeneous solution $\eta$ as in equation (4.3). 
Using (B3) we write (B2) as

$$
Q^{[5]}=\frac{g^{5}}{\pi} \int_{-\infty}^{\infty} d u \int_{-\infty}^{u} d v\left(-\psi_{0} I_{3}^{\delta}+\delta_{1}^{\mathrm{hom}} I_{2}^{\psi}\right)
$$

Using the equations of motion, (4.60) can be written

$$
I_{2}^{\psi}=\frac{1}{2} \partial_{\mu} \psi_{0} \partial^{\mu} \delta_{1}+\frac{4 \psi_{0} \delta_{1}}{(u-v)^{2}}
$$

Similarly,

$$
I_{3}^{\delta}=\frac{1}{4} \partial_{\mu} \delta_{1} \partial^{\mu} \delta_{1}-\frac{1}{2} \delta_{1} \partial_{\mu} \psi_{0} \partial^{\mu} \psi_{0}-\frac{1}{2} \partial_{\mu} \psi_{0} \partial^{\mu} \psi_{2}+\frac{4 \delta_{1}^{2}}{(u-v)^{2}}
$$

Using equations (4.3a), (5.7), (5.8), (B5), and (B6) we can evaluate every piece of (B4) except the part which has the term from (B6) involving $\psi_{2}$. Integrating this term by parts yields

$$
\frac{1}{2} \int_{-\infty}^{\infty} d u \int_{-\infty}^{u} d v \psi_{0} \partial_{\mu} \psi_{0} \partial^{\mu} \psi_{2}=\int_{-\infty}^{\infty} d u \int_{-\infty}^{u} d v \psi_{2} \partial_{u} \partial_{v} \psi_{0}^{2}-\left.\frac{1}{2} \int_{-\infty}^{\infty} d v \psi_{2} \partial_{v} \psi_{0}^{2}\right|_{u=\infty}
$$

Using (5.7) and (B3), the surface term can be written

$$
-\left.\frac{1}{2} \int_{-\infty}^{\infty} d v \psi_{2} \partial_{v} \psi_{0}^{2}\right|_{u=\infty}=\left.\int_{-\infty}^{\infty} d v \psi_{2} \partial_{v} \delta_{1}^{\mathrm{hom}}\right|_{u=\infty}=-\int_{-\infty}^{\infty} d u \int_{-\infty}^{u} d v \delta_{1}^{\mathrm{hom}} I_{2}^{\psi}
$$

Note that this term cancels the second term of (B4). We now examine the first term on the right hand side of (B7). Using (4.8) we find

$$
\begin{gathered}
\int_{-\infty}^{\infty} d u \int_{-\infty}^{u} d v \psi_{2} \partial_{u} \partial_{v} \psi_{0}^{2}=\int_{-\infty}^{\infty} d u \int_{-\infty}^{u} d v \int_{v}^{u} d u^{\prime} \int_{-\infty}^{v} d v^{\prime} F\left(u, v ; u^{\prime}, v^{\prime}\right) I_{2}^{\psi}\left(u^{\prime}, v^{\prime}\right) \\
=\int_{-\infty}^{\infty} d u^{\prime} \int_{-\infty}^{u^{\prime}} d v_{v} \psi_{0}^{2}(u, v) \\
\int_{u^{\prime}}^{\infty} d u \int_{v^{\prime}}^{u^{\prime}} d v F\left(u, v ; u^{\prime}, v^{\prime}\right) \partial_{u} \partial_{v} \psi_{0}^{2}(u, v) \\
I_{2}^{\psi}\left(u^{\prime}, v^{\prime}\right) .
\end{gathered}
$$

We perform the inner $u-v$ integrals as follows. Define the function

$$
\Theta\left(u^{\prime}, v^{\prime}\right)=\int_{u^{\prime}}^{\infty} d u \int_{v^{\prime}}^{u^{\prime}} d v F\left(u, v ; u^{\prime}, v^{\prime}\right) \partial_{u} \partial_{v} \psi_{0}^{2}(u, v)
$$

One can show that 


$$
\left[\partial_{u} \partial_{v}+\frac{2}{(u-v)^{2}}\right] \Theta(u, v)=\partial_{u} \partial_{v} \psi_{0}^{2}=-\frac{1}{2} \partial_{\mu}\left(\psi_{0} \partial^{\mu} \psi_{0}\right)
$$

Hence $\Theta(u, v)$ is a solution to the inhomogeneous equation (B11) with the boundary condition

$$
\lim _{u \rightarrow \infty} \Theta(u, v)=0
$$

Note that the right hand side of (B11) is the same as the right hand side of (4.6a) with $\delta_{0}=-\psi_{0}$. Also, the solution $\psi_{1}$ given by (4.10a) satisfies the condition (B12). Therefore, $\Theta(u, v)$ is given by (4.10a) with $H=-G$ :

$$
\begin{aligned}
\Theta(u, v)= & 2 G^{\prime}(u) G^{\prime}(v)+\frac{6}{(u-v)^{2}}[G(u)-G(v)]^{2} \\
& -\frac{4}{(u-v)^{2}}\left[G^{\prime}(u)+G^{\prime}(v)\right][G(u)-G(v)] .
\end{aligned}
$$

Thus,

$$
\int_{-\infty}^{\infty} d u \int_{-\infty}^{u} d v \psi_{2} \partial_{u} \partial_{v} \psi_{0}^{2}=\int_{-\infty}^{\infty} d u^{\prime} \int_{-\infty}^{u^{\prime}} d v^{\prime} \Theta\left(u^{\prime}, v^{\prime}\right) I_{2}^{\psi}\left(u^{\prime}, v^{\prime}\right)
$$

Using $(\mathbb{B} 6),(\mathbb{B} 7),(\mathbb{B} 8),(\mathbb{B} 13)$, and $(\mathbb{B} 14)$ in $(\mathbb{B} 4)$ and integrating by parts several times we find

$$
Q^{[5]}=\frac{g^{5}}{\pi} \int_{-\infty}^{\infty} d u d v\left[\frac{1}{2} \Theta I_{2}^{\psi}-\frac{3}{2} \frac{\psi_{0} \delta_{1}}{(u-v)^{2}}-\frac{\psi_{0}^{3} \delta_{1}}{(u-v)^{2}}-\frac{1}{8} \frac{\psi_{0}^{5}}{(u-v)^{2}}\right]
$$

which is equation (5.9). In (B15) we found it convenient to use the fact that the integrand is symmetric under the interchange of $u$ and $v$ to extend the integration range over the entire $u-v$ plane. With equation (B15) in hand, we can choose $G(x)$, obtain $\psi_{0}$ from (4.3a), $\delta_{1}$ from (5.8), $I_{2}^{\psi}$ from $(\mathrm{B} 5)$, and $\Theta$ from $(\mathrm{B} 13)$, and then evaluate the fifth order topological charge. 


\section{REFERENCES}

[1] G. 't Hooft, Phys. Rev. D14 (1976) 3432; D18 (1978) 2199.

[2] A. Belavin, A. Polyakov, A. Schwarz, and Yu. Tyupkin, Phys. Lett. B59 (1975) 85.

[3] N. Manton, Phys. Rev. D28 (1983) 2019;

F. Klinkhamer and N. Manton, Phys. Rev. D30 (1984) 2212.

[4] A. Ringwald, Nucl. Phys. B330 (1990) 1;

O. Espinosa, Nucl. Phys. B343 (1990) 310.

For a review, see M. Mattis, Phys. Rep. 214 (1992) 159, and references therein.

[5] V. A. Rubakov, D. T. Son and P. G. Tinyakov, Phys. Lett. B287 (1992) 342; and references therein.

[6] N. H. Christ, Phys. Rev. D21 (1980) 1591.

[7] E. Farhi, V.V. Khoze and R. Singleton, Jr., Phys. Rev. D47 (1993) 5551.

[8] M. Lüscher, Phys. Lett. B70 (1977) 321;

B. Schechter, Phys. Rev. D16 (1977) 3015.

[9] R. Jackiw and C. Rebbi, Phys. Rev. Lett. 37 (1976) 172;

C. Callan, R. Dashen and D. Gross, Phys. Lett. B63 (1976) 334.

[10] E. Witten, Phys. Rev. Lett. 38 (1977) 121;

B. Ratra and L.G. Yaffe, Phys. Lett. B205 (1988) 57. 